\title{
Época e densidade de plantio na produção de cúrcuma.
}

\author{
Time and density of plantation on the turmeric production.
}

\author{
Arthur Bernardes Cecílio Filho ${ }^{1}$ Rovilson José de Souza ${ }^{2}$ \\ Valdemar Faquin ${ }^{3}$ Claudine Márcia de Carvalho ${ }^{4}$
}

\section{RESUMO}

Com o objetivo de avaliar a produtividade da cúrcuma em função da época e densidade de plantio, este experimento foi instalado no período de outubro de 1994 a julho de 1995, em campo, na Universidade Federal de Lavras, no município de Lavras-MG. Foram avaliados os fatores épocas de plantio (20/out, 20/nov, 20/dez e 20/jan) e espaçamento entre plantas na linha de plantio $(0,20 ; 0,35$ e 0,50m), sob delineamento de blocos ao acaso, em parcelas sub-divididas, com três repetições. A produção de rizomas por planta e por área foram influenciados significativamente pela interação dos fatores avaliados. Maiores produtividades por planta $(673,13 \mathrm{~g})$ e por área $\left(24.678,82 \mathrm{~kg} \mathrm{ha}^{-1}\right)$ foram obtidas nos plantios de 20 de novembro e espaçamentos entre plantas, respectivamente, de 0,36 e $0,30 \mathrm{~m}$.

Palavras-chave: Curcuma longa, corante natural, planta medicinal.

\section{ABSTRACT}

With the objective of evaluating the turmeric yield in function of time and plantation density, this experiment was installed in the period of october of 1994 to July of 1995, in field, at Universidade Federal de Lavras, in the city of Lavras$M G$. The factors appraised were plantation times $\left(20^{\text {th }} /\right.$ oct, $20^{\text {th }} /$ nov, $20^{\text {th }} / \mathrm{dec}$ and $\left.20^{\text {th }} / \mathrm{jan}\right)$ and spacing among plants in the plantation line $(0.20 ; 0.35$ and $0.50 \mathrm{~m})$. The experimental design used was randomized blocks, in split plot, with three replications. The production of rhizomes per plant and per area were significantly influenced by the interaction of the appraised factors. Higher production per plant (673.13g) and per area $\left(24,678.82 \mathrm{~kg} \mathrm{ha}^{-1}\right)$ were obtained in the $20^{\text {th }} /$ november planting and spacings among plants, respectively, of 0.36 and $0.30 \mathrm{~m}$.

Key words: Curcuma longa, natural coloring, medicinal plant.

\section{INTRODUÇÃO}

A cúrcuma (Curcuma longa L., família Zingiberaceae), constitui-se numa opção para o crescente mercado de aditivos naturais em alimentos pois, além de sua substância corante, a curcumina, contém óleos essenciais de excelentes qualidades técnicas e organolépticas (DUARTE et al., 1989), que juntos possibilitam estender sua utilização aos mercados de perfumaria, medicinal, alimentício, condimentar, têxtil e outros.

Acredita-se que a Índia seja o principal país produtor, consumidor e exportador. No Brasil, Mara Rosa-GO é o município que apresenta o maior plantio comercial, cuja produção se destina em quase sua totalidade às indústrias nacionais de corantes e alimentos. Entretanto, a exploração feita com baixa tecnologia tem dificultado o crescimento da área de cultivo (GLOBO RURAL, 1994) e, certamente, comprometido a produtividade.

Conforme MARSCHNER (1990), a indução e a taxa de crescimento de órgãos de reserva subterrâneos são fortemente influenciados pelos fatores ambientais.

A definição da melhor época de plantio é função básica da cultura a ser implantada e de fatores climáticos do local, os quais, segundo LEOPOLD \& KRIEDEMANN (1975), MENGEL \& KIRKBY (1987), CASTRO (1987), SALISBURY \& ROSS (1992), afetam vários processos fisiológicos da planta,

${ }^{1}$ Engenheiro Agrônomo, Doutor, Professor Assistente, Departamento de Produção Vegetal da Faculdade de Ciências Agrárias e Veterinárias (FCAV), Universidade Estadual Paulista (UNESP). Via de acesso Prof. Paulo Donato Castellane s/n, 14884-900, Jaboticabal, SP. Email: rutra@fcav.unesp.br Autor para correspondência.

${ }^{2}$ Engenheiro Agrônomo, Doutor, Professor Titular, Departamento de Agricultura, Universidade Federal de Lavras (UFLA), Lavras, MG. ${ }^{3}$ Engenheiro Agrônomo, Doutor, Professor Titular, Departamento de Ciência do Solo, UFLA.

${ }^{4}$ Engenheiro Agrônomo. 
com mudanças qualitativas ou quantitativas no desenvolvimento do vegetal (WENT, 1950; HOLMES \& ROBERTSON, 1959; SQUIRE, 1990).

Não obstante à importância do fator época de plantio, LUCCHESI et al. (1976) e LARCHER (1986) atribuem à densidade de plantio, importante influência sobre a produtividade da cultura. De acordo com HERTWIG (1986), a cúrcuma deve ser plantada de 0,20 a $0,40 \mathrm{~m}$ entre covas e de 0,30 a $0,70 \mathrm{~m}$ entrelinhas de plantio. MARTINS \& RUSIG (1992) recomendam o plantio da cúrcuma em espaçamento de $1,0 \mathrm{~m}$ entrelinhas e $0,40 \mathrm{~m}$ entre plantas na linha, enquanto GOTO (1993) cultivou a cúrcuma em espaçamento de $0,40 \times 0,25 \mathrm{~m}$, apoiando-se em autores indianos, os quais sugerem, para as condições de cultivo da Índia, maiores adensamentos. RAMACHANDRAN \& MUTHUSWAMI (1984) constataram maior rendimento por planta no espaçamento de $0,50 \times 0,225 \mathrm{~m}$ e produção de rizomas por área $90 \%$ superior, no espaçamento de $0,30 \mathrm{x}$ $0,15 \mathrm{~m}$. PHILIP (1985) verificou maior produtividade no espaçamento de $0,25 \times 0,25 \mathrm{~m}$.

Com base no exposto, face a carência de resultados científicos nacionais para subsidiarem o cultivo da cúrcuma, o presente trabalho teve por objetivo determinar as melhores épocas e densidades de plantio da cúrcuma, com base na produtividade da cultura.

\section{MATERIAL E MÉTODOS}

O experimento foi conduzido no período de outubro de 1994 a julho de 1995 na Universidade Federal de Lavras, situada a $21^{\circ} 14^{\prime} \mathrm{S}, 45^{\circ} 00^{\prime} \mathrm{W}$ e altitude média de 910m (CASTRO NETO et al., 1980), com médias anuais de temperatura e precipitação, respectivamente, de $19,4^{\circ} \mathrm{C}$ e $1529,7 \mathrm{~mm}$ (BRASIL, 1992).

Os tratamentos, sob delineamento experimental de blocos ao acaso, em esquema de parcelas sub-divididas, com três repetições, foram constituídos por quatro épocas de plantio (20/outubro, 20/novembro, 20/dezembro e 20/janeiro) nas parcelas e três espaçamentos entre plantas na linha de plantio $(0,20 ; 0,35$ e $0,50 \mathrm{~m})$ nas sub-parcelas, os quais perfazem estandes de 62.500, 35.714 e 25.000 plantas por hectare. $\mathrm{O}$ espaçamento entrelinhas foi de $0,80 \mathrm{~m}$. Cada unidade experimental (sub-parcela) foi constituída por três linhas, sendo considerada área útil para coleta de dados somente a linha central, excluindo-se a primeira e última planta da linha. As áreas total e útil das sub-parcelas, embora tenham contado com o mesmo número de plantas (81), variaram em função do espaçamento entre plantas na linha de plantio e foram, respectivamente, de 12,96 e $4,32 \mathrm{~m}^{2}, 22,68$ e $7,56 \mathrm{~m}^{2}, 32,4$ e $10,8 \mathrm{~m}^{2}$, nos espaçamentos de 0,$20 ; 035$ e $0,50 \mathrm{~m}$ entre plantas.

O plantio foi realizado em leiras de $0,40 \mathrm{~m}$ de altura, colocando-se um rizoma-semente por cova, uniformes quanto ao tamanho, com aproximadamente cinco gramas, colocado a cerca de quatro centímetros de profundidade. $\mathrm{O}$ solo, que não recebeu correção de acidez, apresentava em pré-plantio: $370 \mathrm{~g} \mathrm{~kg}^{-1}$ de argila e $240 \mathrm{~g} \mathrm{~kg}^{-1}$ de areia, $\mathrm{pH}=5,5 ; \mathrm{P}=54 \mathrm{mg} \mathrm{dm}^{-3}$; $\mathrm{K}=180 \mathrm{mg} \mathrm{dm}^{-3}$; Ca, $\mathrm{Mg}$ e Al, 40, 12 e $1 \mathrm{mmol}_{\mathrm{c}} \mathrm{dm}^{-3}$, respectivamente, $38 \mathrm{~g} \mathrm{~kg}^{-1}$ de matéria orgânica, e 44,5; 23,3 e $0,7 \mathrm{mg} \mathrm{kg}^{-1}$ de enxofre, zinco e boro. A adubação de plantio foi de $10,5 \mathrm{~g} /$ planta do fertilizante $\mathrm{N}(4 \%)$ $\mathrm{P}(14 \%)-\mathrm{K}(8 \%)-\mathrm{Ca}(10 \%)-\mathrm{S}(9 \%)$ e a adubação de cobertura foi realizada com $0,84 \mathrm{~g}$ de N/planta, aos 60 dias após emergência das plantas, na fonte sulfato de amônio.

O material genético utilizado foi proveniente de produtores rurais do município de Paracatu (MG) não havendo denominação específica para o germoplasma. Foram realizados tratos culturais necessários à cultura tais como, controle manual (mondas) de plantas daninhas, irrigações (10 a 15mm) a cada dois dias, pelo método de aspersão e reparos nas leiras sempre que necessário. As variáveis avaliadas foram: a) ciclo vegetativo e período de desenvolvimento pós-emergência: expressos em dias, e determinados, respectivamente, pelo período compreendido entre o plantio dos rizomas e emergência das plantas até a colheita final; b) massa seca da parte aérea da planta (MSPA, g planta ${ }^{-1}$ ): mensalmente, foram realizadas coletas de seis plantas competitivas de cada tratamento, para avaliação desta variável. Folhas e pecíolos da planta-mãe e de perfilhos foram lavados e posteriormente, secos em estufa com circulação de ar, a temperatura de $70^{\circ} \mathrm{C}$ até alcançar peso constante, quando se procedeu a pesagem. Foram comparados valores máximos de massa seca da parte aérea produzida, entre os tratamentos avaliados; c) produtividade de rizomas, expressa em kg planta-1 e por kg ha-1).

A colheita final, independente da época de plantio, foi realizada no dia 17 de julho de 1995 , quando as plantas apresentavam-se com a parte aérea completamente seca, induzida por baixas temperaturas do inverno.

A análise estatística dos dados foi realizado no programa SANEST, com a análise de regressão dos dados quando os fatores foram significativos a $5 \%$ de probabilidade de erro pelo Teste F. As equações de regressão determinadas no mesmo Programa para 
representarem a resposta da cúrcuma segundo os tratamentos avaliados, foram escolhidas segundo o melhor ajuste $\left(\mathrm{r}^{2}\right)$ e menor probabilidade de erro.

\section{RESULTADOS E DISCUSSÃO}

Foi observado efeito significativo somente do fator época de plantio para ciclo e período de desenvolvimento pós emergência. $\mathrm{O}$ ciclo de cultivo foi tanto maior quanto mais cedo realizou-se o plantio (Figura 1). Para os plantios realizados em 20/out, 20/ nov, 20/dez e 20/jan, os ciclos foram de 270, 240, 210 e 180 dias, respectivamente. Embora PURSEGLOVE (1972) e HERTWIG (1986) citem a cúrcuma como espécie perene, as condições climáticas do município de Lavras a torna metabolicamente anual, sendo colhida sempre no mês de julho, quando sua parte aérea senesce em decorrência das baixas temperaturas.

Quanto ao período de desenvolvimento pós emergência (PDPE), a linearidade da resposta constatada para extensão do ciclo de cultivo, neste caso, não foi verificada, devido à diferença no comprimento do período de emergência das plântulas de cúrcuma entre as épocas de plantio. O período de emergência foi de 40 e 15 dias, respectivamente, para os plantios realizados em 20/out e 20/nov e de 12 dias para os plantios nas demais épocas avaliadas, resultando assim, num comportamento quadrático do PDPE (Figura 1). Atribui-se ao longo período para a emergência das plantas no plantio em 20/out à dormência, uma vez que foi a única época de plantio em que os rizomas-sementes não se apresentavam brotados.

Houve interação significativa dos fatores para produção máxima de massa seca de parte aérea (MSPA). O máximo de MSPA ocorreu no plantio de 20/nov e espaçamento de $37,7 \mathrm{~cm}$ entre plantas, alcançando 50,06g de massa seca por planta (Figura 2). Esta quantidade foi superior em $106 \%, 137 \%$ e $212 \%$ aos valores máximos observados, respectivamente, nos plantios de 20/out, 20/dez e 20/ jan e em $169 \%$ e $43 \%$ aos valores observados, respectivamente, nos espaçamentos de 20 e $50 \mathrm{~cm}$

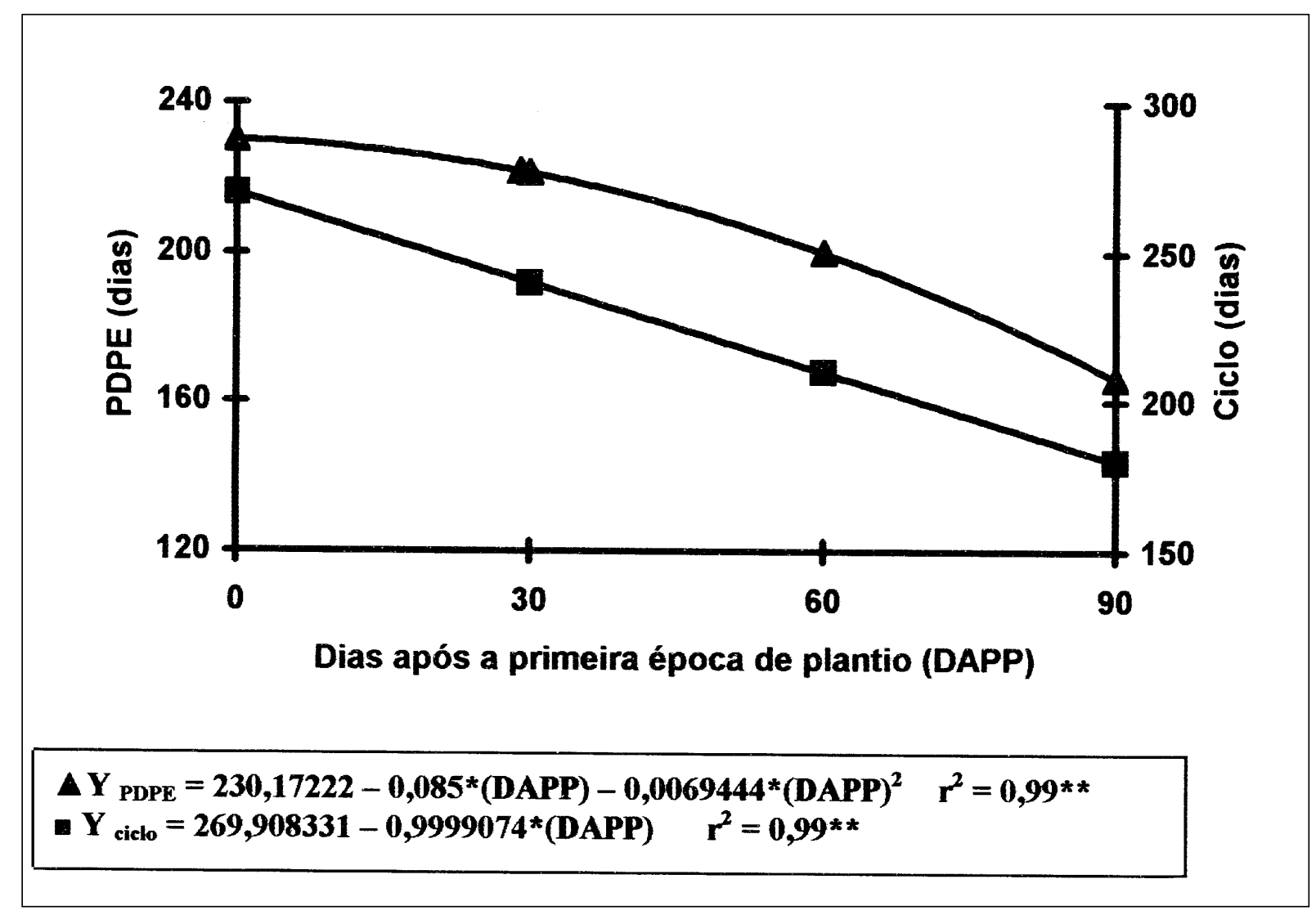

Figura 1.- Influência da época de plantio (0, 30, 60 e 90 dias após o primeiro plantio - DAPP - correspondem, respectivamente, aos plantios de 20/out, 20/nov, 20/dez e 20/jan) sobre a extensão do ciclo e período de desenvolvimento pós emergência da cúrcuma (PDPE).

Ciência Rural, v. 34, n.4, jul-ago, 2004. 


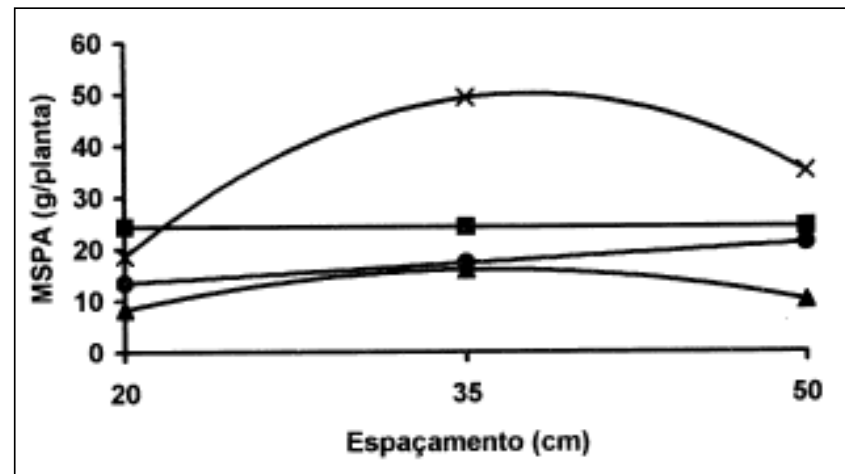

- Yout $=$ n.s.

X Ynev $=-92,335552+7,5471109^{*}(\mathrm{~cm})-0,1^{*}(\mathrm{~cm})^{2} \quad r^{2}=1,0^{* *}$

- Ydez $=8,273889+0,257667^{*}(\mathrm{~cm}) \quad r^{2}=0,80^{*}$

$\Delta Y j a n=-23,527407+2,1974074^{\star}(\mathrm{cm})-0,03051852^{*}(\mathrm{~cm})^{2} \quad r^{2}=1,0^{\star}$

Figura 2 - Massa seca da parte aérea (MSPA) de plantas de cúrcuma, nos plantios de 20/out, 20/nov, 20/dez e 20/jan, em função dos espaçamentos entre plantas na linha de plantio.

entre plantas, no próprio plantio de 20/nov. Considerando-se graus-dia conforme definição de HOLMES \& ROBERTSON (1959), em que um graudia representa o calor efetivo para o crescimento da planta acumulado no dia, verificou-se a disponibilidade de 2425, 2053, 1831 e 1501 grausdia, para os plantios em 20/out, 20/nov, 20/dez e 20/ jan, adotando-se como $10^{\circ} \mathrm{C}$ a temperatura base da cúrcuma, proposta por GOTO (1993). Entretanto, é importante salientar que no plantio de 20/out., embora tenha havido maior disponibilidade de calor efetivo para o crescimento da planta, observou-se também, um longo período para emergência, fato que não possibilitou o aproveitamento pela cúrcuma de todo o calor disponível para seu efetivo desenvolvimento vegetativo.

Paralelamente, foi observado que o aumento da densidade de plantio (espaçamentos menores que $50 \mathrm{~cm}$ entre plantas na linha), possibilitou incremento no acúmulo de MSPA até o espaçamento de $37,7 \mathrm{~cm}$, tido como ponto de competição, conforme MENDOZA (1982) e CHOAIRY \& FERNANDES (1983). À medida em que se continuou a reduzir o espaçamento entre plantas, menores foram os valores de MSPA, verificando-se redução de até $68 \%$ da massa seca produzida em relação ao melhor tratamento.

A produção de rizomas por planta mostrou ser dependente da interação $(\mathrm{p}<0,01)$ entre os fatores estudados. Maior produção, $673,13 \mathrm{~g} \mathrm{planta}^{-1}$, foi obtida no plantio de $20 /$ nov, no espaçamento de $36,6 \mathrm{~cm}$ (Figura 3). Este valor foi superior em $41 \%$ ao máximo verificado no plantio de $20 /$ out e espaçamento de $37,7 \mathrm{~cm}$ $\left(476,99 \mathrm{~g} \mathrm{planta}^{-1}\right)$ e em $170,8 \%$ e $277 \%$ aos valores médios observados, respectivamente, nos plantios de 20/dez $(248,50 \mathrm{~g})$ e $20 /$ jan $(178,5 \mathrm{~g})$, refletindo o comportamento descrito para produção de MSPA. Os resultados observados concordam com trabalhos de JHA et al. (1983) e RANDHAWA et al. (1984), os quais constataram que o atraso no plantio resulta em menor período de crescimento da parte aérea e enchimento dos rizomas.

Quanto à produção de rizomas por hectare, também foi observado interação significativa dos fatores em avaliação. A maior produtividade $\left(24.678,82 \mathrm{~kg} \mathrm{ha}^{-1}\right)$ foi obtida no plantio de $20 /$ nov e espaçamento entre plantas de aproximadamente $30 \mathrm{~cm}(30,3 \mathrm{~cm})$, sendo superior em $20 \%, 88 \%$ e $148 \%$ às maiores produtividades obtidas, respectivamente, nos plantios de 20/out, 20/dez e 20/jan (Figura 4), condição esta que também proporcionou o maior índice de área foliar $(2,3)$.

Nota-se que a maior produtividade $\left(24.678,82 \mathrm{~kg} \mathrm{ha}^{-1}\right)$ obtida no presente trabalho, com densidade de 41.666 plantas ha $^{-1}$, encontrase muito próxima às produtividades de rizomas conseguidas na Índia, como por exemplo, $22.910 \mathrm{~kg}$

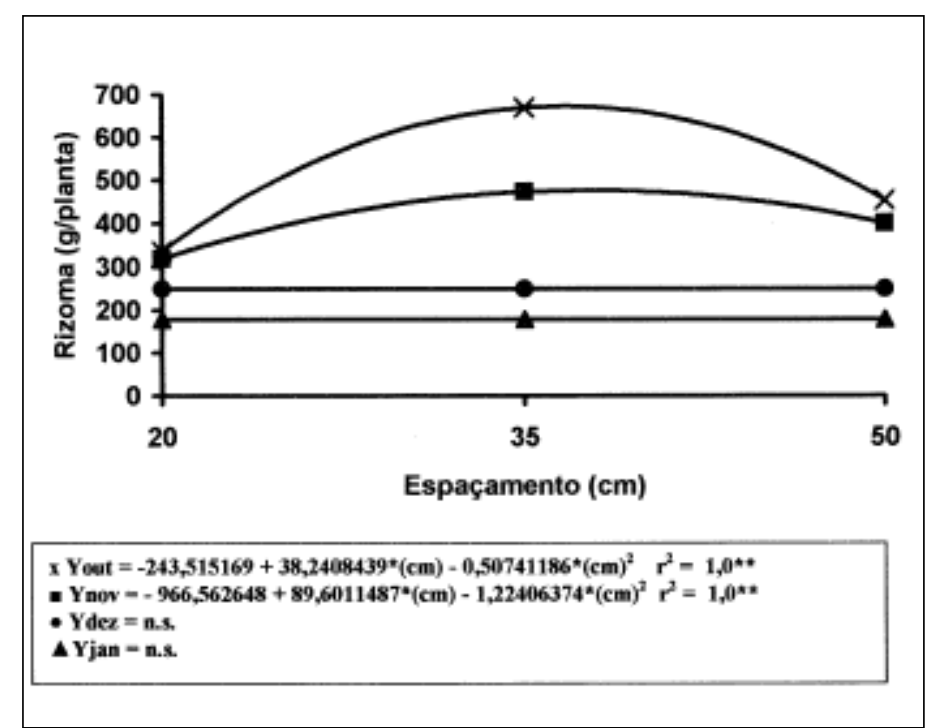

Figura 3- Produção de rizomas de cúrcuma, em g/planta, nos plantios de 20/out, 20/nov, 20/dez e 20/jan, em função dos espaçamentos entre plantas na linha de plantio. 


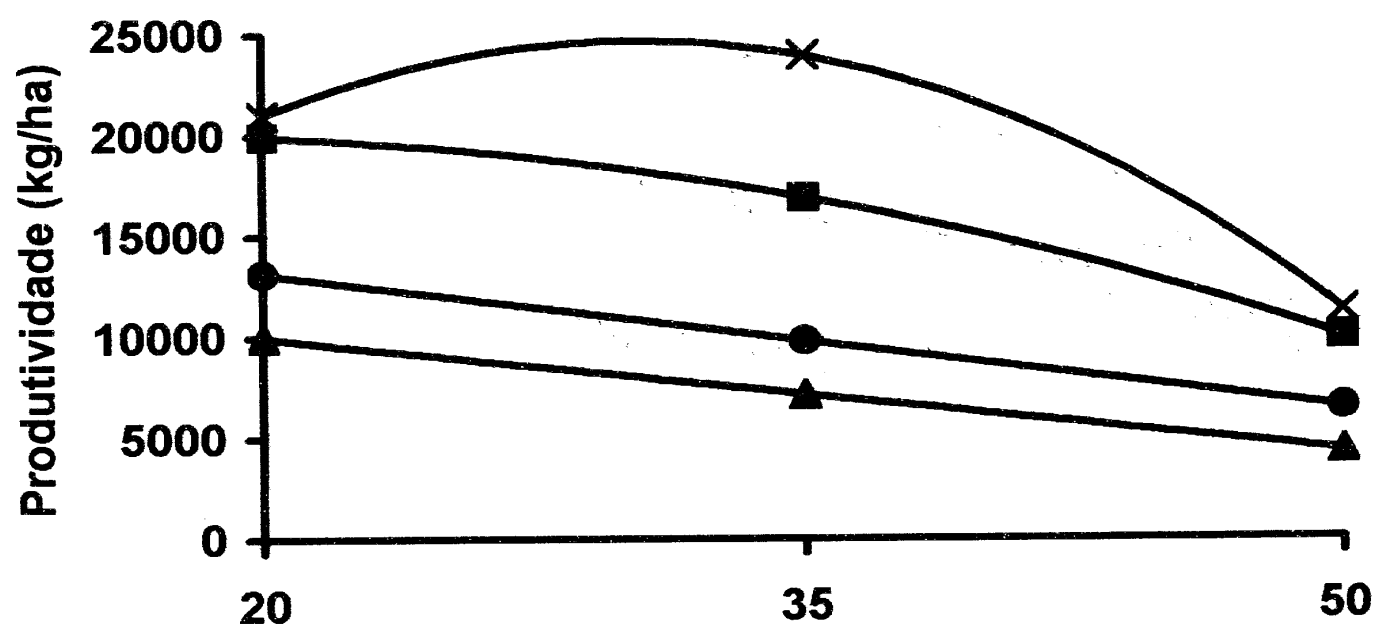

Espaçamento (dias)

x Yout $=17796,512876+278,9164099 *(\mathrm{~cm})-8,69696108 *(\mathrm{~cm})^{2} \quad r^{2}=1,0 *$

- Ynov $=-7092,894387+2094,530259 *(\mathrm{~cm})-34,52009404^{*}(\mathrm{~cm})^{2} \quad \mathbf{r}^{2}=1,0^{\star}$

- Ydez $=17514,817356-219,9053765^{*}(\mathrm{~cm}) \quad \mathbf{r}^{2}=0,99^{* *}$

$\triangle$ Yjan $=13650,96877-185,1908203^{*}(\mathrm{~cm}) \quad \mathbf{r}^{2}=0,99 * *$

Figura 4 - Produtividade de rizomas de cúrcuma, em kg ha ${ }^{-1}$, nos plantios de 20/out, 20/nov, 20/dez e 20/jan, em função dos espaçamentos entre plantas na linha de plantio.

$\mathrm{ha}^{-1}$ (SHAH \& MUTHUSWAMI, 1981) e de $28.125 \mathrm{~kg} \mathrm{ha}^{-1}$ (PHILIP, 1983), na média de 6.632 a $50.086 \mathrm{~kg} \mathrm{ha}^{-1}$ observadas por PHILIP \& NAIR (1983), sempre com densidades superiores a 110.000 plantas $\mathrm{ha}^{-1}$.

\section{CONCLUSÃO}

O plantio da cúrcuma com rizomassemente sem brotos desenvolvidos, acarreta em longo período de emergência, com reflexos negativos sobre a produção de massa seca da parte aérea e produtividade da planta.

Os fatores época e densidade de plantio afetam significativamente o potencial produtivo (MSPA) da planta.

A produtividade da cultura da cúrcuma é influenciada significativamente pela época e densidade de plantio, sendo maximizada no plantio em 20 de novembro e espaçamento de $0,30 \mathrm{~m}$ entre plantas na linha de plantio e $0,80 \mathrm{~m}$ entrelinhas.

\section{REFERÊNCIAS BIBLIOGRÁFICAS}

BRASIL. Ministério da Agricultura. Departamento Nacional de Meteorologia. Normais Climatológicas - 1961 a 1990. Brasília, 1992. $84 \mathrm{p}$.

CASTRO, P.R.C. Ecofisiologia da produção agrícola. Piaracicaba : Potafos, 1987. 249p.

CASTRO NETO, P; SEDIYAMA, G.C.; VILELA, E de A. Probabilidade de ocorrência de períodos chuvosos em Lavras, Minas Gerais. Ciência e Prática, v.4, n.1, p.56-65, jan/jun, 1980.

CHOAIRY, S.A.; FERNANDES, P.D. Densidades de plantio na cultura do abacaxi. Pesquisa Agropecuária Brasileira, v.18, n.9, p.985-988, set, 1983.

DUARTE, R.D.; BOVI, O.A.; MAIA, N.B. Corantes - Programa de pesquisa do Instituto Agronômico de Campinas. In: SEMINÁRIO DE CORANTES NATURAIS PARA ALIMENTOS, 1., 1989, Campinas, SP. Anais... Campinas : ITAL, 1989. p.45-53.

GLOBO RURAL. Açafrão - O ouro da cozinha. Rio de Janeiro: Globo, dez, 1994. n.110. p.38-43.

GOTO, R. Épocas de plantio, adubação fosfatada e unidades térmicas em cultura de açafrão (Curcuma longa L.). 1993. 93f. Tese (Doutorado em Produção Vegetal) - Curso de Pósgraduação em Agronomia, Produção Vegetal, Faculdade de Ciências Agrárias e Veterinárias, Universidade Estadual Paulista.

Ciência Rural, v. 34, n.4, jul-ago, 2004. 
HERTWIG, I.F.Von. Curcuma. In: HERTWIG, I.F.Von. Plantas aromáticas e medicinais. São Paulo : Icone, 1986. p.254-265.

HOLMES, R.M.; ROBERTSON, G.W. Heats units and crop growth. Ottawa : Canada Departament of Agriculture, 1959. p.35.

JHA, R.C.; SHARMA, N.N.; MAURYA, K.R. Effect of sowing dates and mulching on the yield and profitability of turmeric (Curcuma longa L.). Bangladesh Horticulture, v.11, n.1, p.14. 1983.

LARCHER, W. Utilização de carbono e produção de matéria seca. In: LARCHER, W. Ecofisiologia vegetal, São Paulo : EPU, 1986. p.74-160.

LEOPOLD, A.C.; KRIEDEMANN, P.E. Plant growth and development. New York : Mc Graw-Hill, 1975. 545p.

LUCCHESI, A.A. et al. Produtividade do rabanete (Raphanus sativus L.) relacionado com a densidade de população. Anais da Escola Superior de Agricultura Luiz de Queiroz, v.33, p.577-83.1976.

MARSCHNER, H. Mineral nutrition of higher plants. London: Academic, 1990. 674p.

MARTINS, M.C.; RUSIG, O. Cúrcuma: um corante natural. Boletim da Sociedade Brasileira de Ciência e Tecnologia de Alimentos, Campinas, v.26, n.1, p.56-65, 1992.

MENDONZA, J.F.B. Efeitos de poda e população de tomate (Lycopersicon esculentum Mill). In: MULLER,J.J.V.; CASALI,V.W.D. Seminários de olericultura. Viçosa : UFV, 1982. V.4, p.122-140.

MENGEL, K.; KIRKBY, E.A. Principles of plant nutrition. Bern : International Potash Institute, 1987. $687 \mathrm{p}$.
PHILIP, J. Studies on growth, yield and quality components in different turmeric types. Indian Cocoa, Arecanut and Spices Journal, v.6, n.4, p.93-97, 1983.

PHILIP, J. Effect of plant density on yield and yield components of turmeric. Indian Cocoa, Arecanut and Spices Journal, v.8, n.4, p.93-96, 1985.

PHILIP, J; NAIR, P.C.S. Morphological and yield characters of turmeric types. Indian Cocoa, Arecanut and Spices Journal, v.7, n.3, p. 61-67, 1983 .

PURSEGLOVE, J.W. Zingiberaceae. In: PURSEGLOVE, J.W. Tropical crops - Monocotyledons. London : Willian Cloves \& Sons, v.2, p.519-544, 1972.

RAMACHANDRAN, M.; MUTHUSWAMI, S. Studies on the influence of method of planting and spacing on yield and quality of turmeric. South Indian Horticulture, v.32, n.3, p.143-145, 1984.

RANDHAWA, G.S. et al. Performance of turmeric (Curcuma longa L.) under different dates and methods of sowing. Journal Research Punjab Agricultural University, v.21, n.4, p.489-495, 1984.

SALISBURY, F.B.; ROSS, C. Plant physiology. Belmont: Wadsworth, 1992. p.425-443.

SHAH, H.A.; MUTHUSWAMI, S. Studies on the influence of nitrogen on the yield and yield components of turmeric (Curcuma longa L.). South Indian Horticulture, v.29, n.1, p.9-10. 1981.

SQUIRE, G.R. The physiology of tropical crop production. Wallingford : CAB International, 1990. 236p.

WENT, F.W. The response of plants to climate. Science, v.112, n.1/2, p.489-494, 1950. 